\title{
Soluble urokinase plasminogen activator receptor and hypertension among black South Africans after 5 years
}

\begin{abstract}
Shani Botha ${ }^{1}$, Carla MT Fourie ${ }^{1}$, Rudolph Schutte ${ }^{1}$, Jesper Eugen-Olsen ${ }^{2}$ and Aletta E Schutte ${ }^{1}$
Soluble urokinase plasminogen activator receptor (suPAR) is a biomarker that links inflammation with cardiovascular risk. However, studies linking suPAR and hypertension are scant. First, we determined whether baseline suPAR is elevated in normotensive black South Africans who developed hypertension over 5 years, compared with those who remained normotensive; and second, whether hypertension is associated with suPAR. This substudy is embedded in the South African leg of the Prospective Urban and Rural Epidemiology study, performed in the North West Province. We investigated 429 normotensive individuals, of which 191 developed hypertension and 238 remained normotensive over 5 years. We determined suPAR from plasma (ethylenediaminetetraacetic acid) samples with the suPARnostic ELISA Kit and blood pressure with an OMRON HEM-757 device. Despite similar mean baseline suPAR levels $(P=0.43)$, suPAR increased more in the group that developed hypertension compared with those who remained normotensive $(14.2 \%$ vs. $6.94 \% ; P=0.007)$. Five-year percentage change in systolic blood pressure correlated positively $(r=0.23 ; P=0.002)$ and associated independently with baseline suPAR $(\beta=0.14$; $P=0.043)$, only in participants who developed hypertension. Participants were 1.41 times more likely $(P=0.015)$ to develop hypertension with 1 s.d. increase in percentage change in suPAR levels over 5 years. Change in systolic blood pressure was associated with baseline suPAR in hypertensive participants and change in suPAR with hypertensive status. This study highlights the need for more research on the role of suPAR in hypertension and cardiovascular disease development in black South
\end{abstract} Africans.

Hypertension Research (2015) 38, 439-444; doi:10.1038/hr.2015.22; published online 5 March 2015

Keywords: African; blood pressure; epidemiology; inflammation; South Africa

\section{INTRODUCTION}

Inflammation forms an integral part of the mechanisms involved in the development of cardiovascular and metabolic diseases and has received attention as a factor mediating both the genesis and the development of hypertension. ${ }^{1-3}$

In a hypertensive milieu, factors such as excessive mechanical stress increase oxidative stress, impair endothelial function and lead to atherosclerosis. ${ }^{4}$ It is further known that, in hypertensive patients, vascular endothelial cells, monocytes and activated $\mathrm{T}$ cells are especially activated and produce various cytokines. ${ }^{2,5}$ The urokinase plasminogen activator receptor, for example, is expressed by such cell types ${ }^{6}$ and soluble urokinase plasminogen activator receptor (suPAR), a marker of inflammation, ${ }^{7}$ is formed during inflammatory stimuli when the receptor is cleaved from the cellular membrane. ${ }^{8}$

SuPAR is associated with type $1^{9}$ and 2 diabetes, incident cancer, cardiovascular disease and total mortality in the general population. ${ }^{7}$ More recently, a large study involving 3367 subjects (67\% with coronary artery disease) showed that elevated levels of plasma suPAR are associated with the presence and severity of coronary artery disease, as well as that suPAR is an independent predictor of death and myocardial infarction. ${ }^{10,11}$ SuPAR further predicts cardiovascular events ${ }^{10,11}$ and provides additional prognostic information over and beyond the Framingham Risk Score, especially when combined with C-reactive protein (CRP). ${ }^{12}$ There is however a paucity of information regarding the role of suPAR in hypertension, especially among the understudied black South African population in which the prevalence of hypertension is very high. ${ }^{13}$

First, we investigated baseline and change in suPAR levels in black South Africans who developed hypertension and in those who remained normotensive after 5 years; and second, whether hypertension is associated with suPAR.

\section{METHODS}

Study design and participants

This substudy is embedded in the international Prospective Urban and Rural Epidemiology (PURE) study. ${ }^{14}$ We investigated 1068 HIV-uninfected black men and women from urban and rural areas in the North West Province, South Africa. Data collection for this substudy took place two times, namely in 2005 (where 520 normotensive participants were included; Supplementary Figure S1) and in 2010. From these normotensive participants, 191 (36.7\%)

${ }^{1}$ Hypertension in Africa Research Team (HART), North-West University, Potchefstroom, South Africa and ${ }^{2}$ Clinical Research Centre 136 , Copenhagen University Hospital, Hvidovre, Denmark

Correspondence: S Botha, Hypertension in Africa Research Team (HART), North-West University, Private Bag X6001, Potchefstroom 2520, South Africa.

E-mail: shani.botha@nwu.ac.za

Received 6 November 2014; revised 28 January 2015; accepted 3 February 2015; published online 5 March 2015 
developed hypertension over 5 years and 238 remained normotensive (excluding 73 participants who were using antihypertensive, anti-inflammatory, antidiabetic and statin medication, and excluding 18 who had incomplete data at follow-up). The exact date on which participants became hypertensive during the 5-year interval could, however, not be determined.

The study protocol was approved by the Ethics Committee of the NorthWest University in South Africa and complied with the Declaration of Helsinki (as revised in 2004). Informed consent was obtained from each participant. A detailed layout of the experimental protocol for data collection was described previously. ${ }^{15}$

\section{Questionnaires}

Specially trained field workers obtained questionnaire data in the participants' home language. Such data included demographic and lifestyle information such as locality, gender, age, education, employment, alcohol consumption, tobacco and medication use.

\section{Anthropometric and cardiovascular measurements}

We used standardized procedures to obtain anthropometric measurements, including height (Invicta Stadiometer, IP 1465; Invicta, London, UK), weight
(Precision Health Scale; A \& D Company, Tokyo, Japan), hip and waist circumference (Holtain unstretchable flexible $7 \mathrm{~mm}$ wide metal tape; Crosswell, Pembrokeshire, Wales). ${ }^{16}$

Using an appropriate cuff size, a trained observer took brachial systolic (SBP) and diastolic blood pressure with an OMRON HEM-757 device (Omron Healthcare, Kyoto, Japan), in duplicate, 5-min apart, whereas the participant was in the sitting position. The second value was used. We defined hypertension according to the $2013 \mathrm{ESH} / \mathrm{ESC}$ guidelines as an SBP $\geqslant 140$ $\mathrm{mm} \mathrm{Hg}$ and/or diastolic blood pressure $\geqslant 90 \mathrm{~mm} \mathrm{Hg} .{ }^{17}$

\section{Biochemical analyses}

After we obtained fasting blood samples, serum and plasma were prepared according to appropriate methods. These samples were snap frozen on dry ice and then stored at $-80^{\circ} \mathrm{C}$ in the laboratory until analysis. SuPAR was determined from plasma (ethylenediaminetetraacetic acid) samples with the suPARnostic ELISA Kit (ViroGates, Copenhagen, Denmark). ${ }^{18}$ A Konelab20i ${ }^{\mathrm{TM}}$ autoanalyzer (Thermo Fisher Scientific Oy, Vantaa, Finland) was used in 2005 and a Cobas Integra 400 Roche Clinical System (Roche Diagnostics, Indianapolis, IN, USA) in 2010 to determine total cholesterol, high-density lipoprotein cholesterol, triglycerides, $\gamma$-glutamyltransferase and creatinine (by means of an

Table 1 Characteristics of black South African participants showing differences in baseline (2005) and follow-up (2010) of participants who remained normotensive $(n=238)$ and those who developed hypertension $(n=191)$ after 5 years, respectively

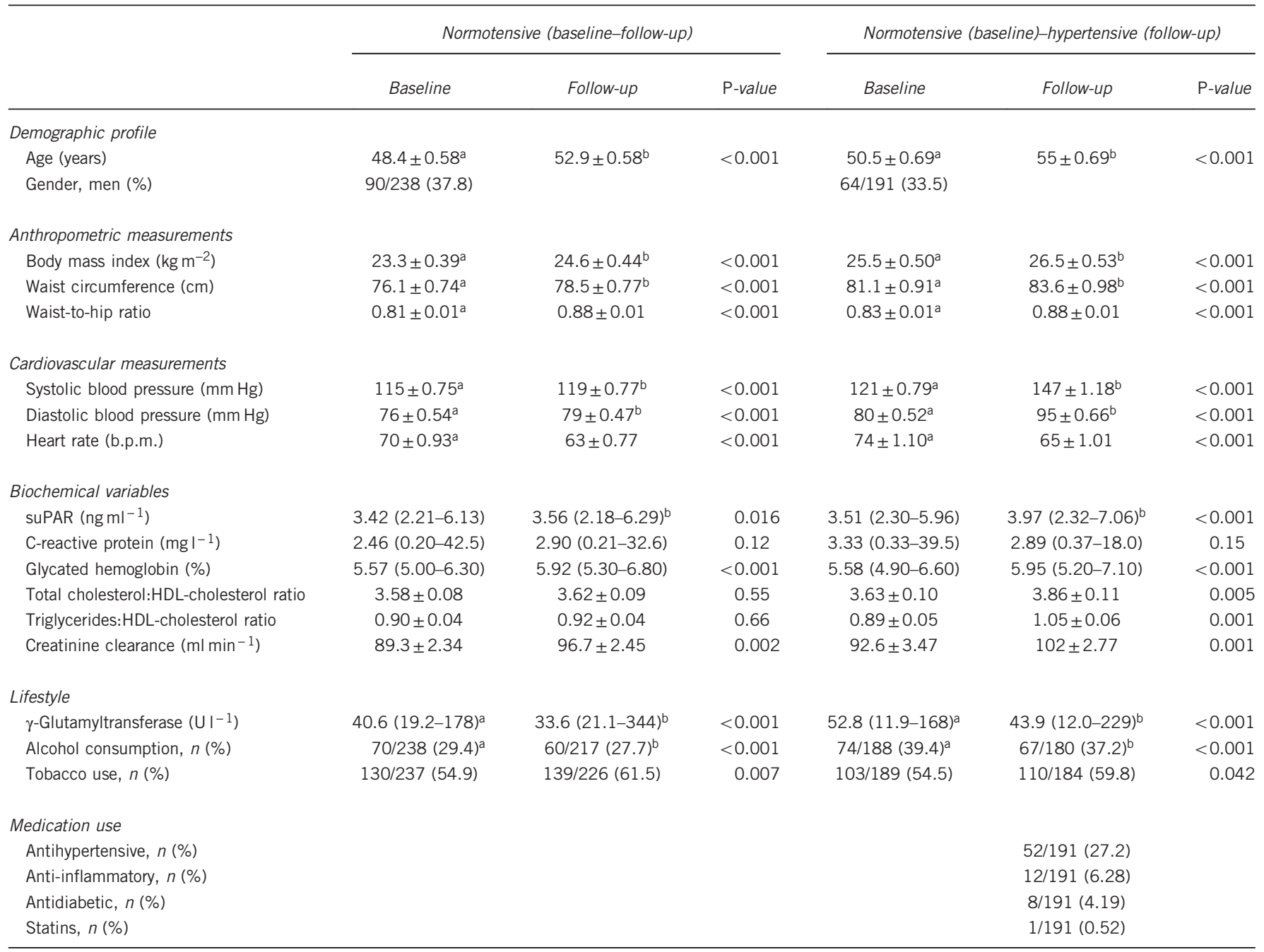

Abbreviations: HDL, high-density lipoprotein; suPAR, soluble urokinase plasminogen activator receptor.

Data are expressed as arithmetic mean \pm s.e., geometric mean (5th and 95 th percentile boundaries) or $\%$ of $n$. P-values for comparison between groups were obtained with dependent $t$-tests and Data are expressed as arithmetic mean \pm s.e., geometric mean
McNemar tests.

aStatistical significant difference between groups at baseline

bStatistical significant difference between groups at follow-up. $P \leqslant 0.05$ was regarded as statistically significant. 
enzymatic colorimetric method), as well as high-sensitivity CRP (by means of a particle enhanced turbidimetric assay) from serum samples. Glucose was determined in sodium fluoride tubes by means of an enzymatic reference method with hexokinase (Vitros DT6011 Chemistry Analyzer (Ortho-Clinical Diagnostics, Rochester, NY, USA) in 2005 and Cobas Integra 400 Roche Clinical System (Roche Diagnostics, Indianapolis, IN, USA) in 2010). Glycated hemoglobin was determined from whole blood (ethylenediaminetetraacetic acid) samples, based on ion-exchange high-performance liquid chromatography, with the D-10 Hemoglobin Testing System from Bio-Rad (Hercules, CA, USA; no. 220-0101). Intra- and intercoefficients of variation for all assays were below $10 \%$. We determined estimated creatinine clearance using the Cockcroft-Gault formula. 19

\section{Statistical analyses}

Variables with a skewed distribution were logarithmically transformed (suPAR, CRP, plasminogen activator inhibitor-1, glycated hemoglobin and glucose). We compared means and proportions with independent $t$-tests and $\chi^{2}$ tests, respectively. Adjusted differences were determined with analyses of covariance, whereas differences in means and proportions between baseline and follow-up within each group were determined with dependent $t$-tests and McNemar tests, respectively. We established relationships between variables with Pearson's correlations, while performing partial correlations and multiple linear regression analyses (forward stepwise) to establish independent relationships. In the latter, we used percentage change in SBP (\%SBP) as the dependent variable, with baseline and percentage change in suPAR (\%suPAR) as the main independent variables. Other covariates that were entered into the models were chosen based on the literature ${ }^{17,20}$ and on exploratory partial correlation analyses. We further performed a logistic regression analysis to determine odds ratios, with hypertension status as dependent variable and \%suPAR as independent variable. The analysis was repeated, while adjusting for other covariates. Percentage change was calculated as the difference between followup and baseline values, divided by the baseline value and multiplied by 100 . Statistical analyses were performed with Statistica version 12.0 (Statsoft, Tulsa, OK, USA)

\section{RESULTS}

Of the 520 black South Africans who were normotensive at baseline, we included 429 , of which $35 \%$ were men, $61 \%$ were situated in rural areas, $21 \%$ were employed and $58 \%$ had at least a primary school education. In these participants, suPAR related strongest to age, CRP, $\gamma$-glutamyltransferase and tobacco use (all $P<0.009$ ) (Supplementary Table S1). Table 1 summarises the baseline and follow-up

Table 2 Change in characteristics of black South African participants over five years

\begin{tabular}{lccc} 
& $\begin{array}{c}\text { Normotensive at } \\
\text { follow-up }(\mathrm{n}=238)\end{array}$ & $\begin{array}{c}\text { Hypertensive at } \\
\text { follow-up }(\mathrm{n}=191)\end{array}$ & P-value \\
\hline $\begin{array}{l}\text { Percentage change (\%) } \\
\text { Waist circumference }\end{array}$ & $3.26 \pm 0.43$ & $3.38 \pm 0.57$ & 0.86 \\
Systolic blood pressure & $3.63 \pm 0.78$ & $22.0 \pm 1.15$ & $<0.001$ \\
Diastolic blood & $4.92 \pm 0.92$ & $19.4 \pm 1.10$ & $<0.001$ \\
pressure & & & \\
suPAR & $6.94 \pm 1.76$ & $14.2 \pm 2.05$ & 0.007 \\
$\begin{array}{l}\text { C-reactive protein } \\
\text { Glycated hemoglobin }\end{array}$ & $132 \pm 23.2$ & $73.1 \pm 18.4$ & 0.055 \\
$\begin{array}{l}\text { Triglycerides: } \\
\text { HDL-cholesterol ratio }\end{array}$ & $18.2 \pm 5.00$ & $7.60 \pm 0.79$ & 0.21 \\
$\gamma$-Glutamyltransferase & $-9.48 \pm 3.09$ & $27.1 \pm 5.77$ & 0.25 \\
\end{tabular}

Abbreviations: HDL, high-density lipoprotein; suPAR, soluble urokinase plasminogen activator receptor.

Data are expressed as arithmetic mean + s.e. $P$-values for comparison between normotensive and hypertensive groups were obtained with independent $t$-tests. $P \leqslant 0.05$ was regarded as statistically significant characteristics according to hypertensive status. Anthropometric measurements, systolic and diastolic blood pressure, glycated hemoglobin, creatinine clearance and tobacco use increased (all $P \leqslant 0.002$ ), whereas heart rate and measures of alcohol consumption decreased (all $P \leqslant 0.042$ ) in both those who remained normotensive and those who became hypertensive after 5 years. At follow-up, the 191 participants who became hypertensive were older $(P=0.021)$, had a higher alcohol consumption $(P=0.042), \gamma$-glutamyltransferase levels $(P=0.003)$, body mass index $(P=0.006)$ and waist circumference measure $(P<0.001)$, whereas no differences in tobacco use $(P=0.72)$ were seen. SBP increased $18 \%$ more $(P<0.001)$ in participants who became hypertensive than those who remained normotensive (Table 2). Of the 191 hypertensives at follow-up, 27\% were hypertensive despite being treated with antihypertensive medication (Table 1).

Although baseline suPAR did not differ $(P=0.43)$ between those who became hypertensive and remained normotensive, suPAR increased more in hypertensive participants (14.2\% vs. 6.94\%; $P=0.007)$ and was higher at follow-up compared with the normotensive group $(P=0.002)$. This was not the case for CRP at baseline $(P=0.070)$ or follow-up $(P=0.84)$ (Tables 1 and 2$)$.

In univariate analyses, $\% \mathrm{SBP}$ correlated positively with baseline suPAR, but only in the hypertensive group $(r=0.23 ; P=0.002)$ (Figure 1). \%SBP did however not correlate with \%suPAR in either the hypertensive $(r=-0.11 ; P=0.14)$ or the normotensive $(r=-0.06$; $P=0.42$ ) groups (not shown). \%SBP increased with an increase in baseline suPAR tertiles in those who developed hypertension ( $P$-trend $=0.030)$, but not in those who remained normotensive $(P$-trend $=0.98)$ (Supplementary Figure S2). Again, this was not the case when tertiles of \%suPAR was used. We confirmed these findings in multivariate-adjusted analyses (Table 3) where \%SBP correlated positively with baseline suPAR only in the hypertensive group $(\beta=0.14 ; P=0.043)$.

We determined standardised odds ratios with hypertension status as dependent variable and \%suPAR as independent variable. In bivariate analyses, we found that participants were 1.32 times $(P=0.008)$ more likely to become hypertensive over 5 years with each standard deviation increase in suPAR. These results also remained significant (odds ratio $=1.41 ; P=0.015$ ) in a multivariate analysis (Table 4 ).

\section{DISCUSSION}

We investigated the inflammatory marker suPAR and associations thereof with hypertension in black South Africans. SuPAR increased over 5 years in both groups, but more prominently in those who developed hypertension. \%SBP independently associated with baseline suPAR, only in the group that developed hypertension, whereas, after multivariate adjustment, a 1 s.d. increase in \%suPAR was associated with a $41 \%$ more likely risk to be hypertensive.

Even though suPAR relates to renal disease, ${ }^{21}$ cancer, type 1 and 2 diabetes, ${ }^{7,9}$ cardiovascular disease and mortality, ${ }^{7}$ limited supportive evidence exist with regard to the positive relationship of suPAR with hypertension per se.

Hypertension is currently well known as an inflammatory disease ${ }^{2}$ and increased levels of various inflammatory biomarkers precede the development of hypertension. ${ }^{3,22-24}$ We found that, even though both suPAR and blood pressure increased over 5 years, \%suPAR did not directly associate with change in SBP, whereas baseline suPAR did. SuPAR levels were, however, similar for both groups at baseline and \% suPAR did associate with being hypertensive. The controversy in our findings give rise to the question whether suPAR is involved in the 


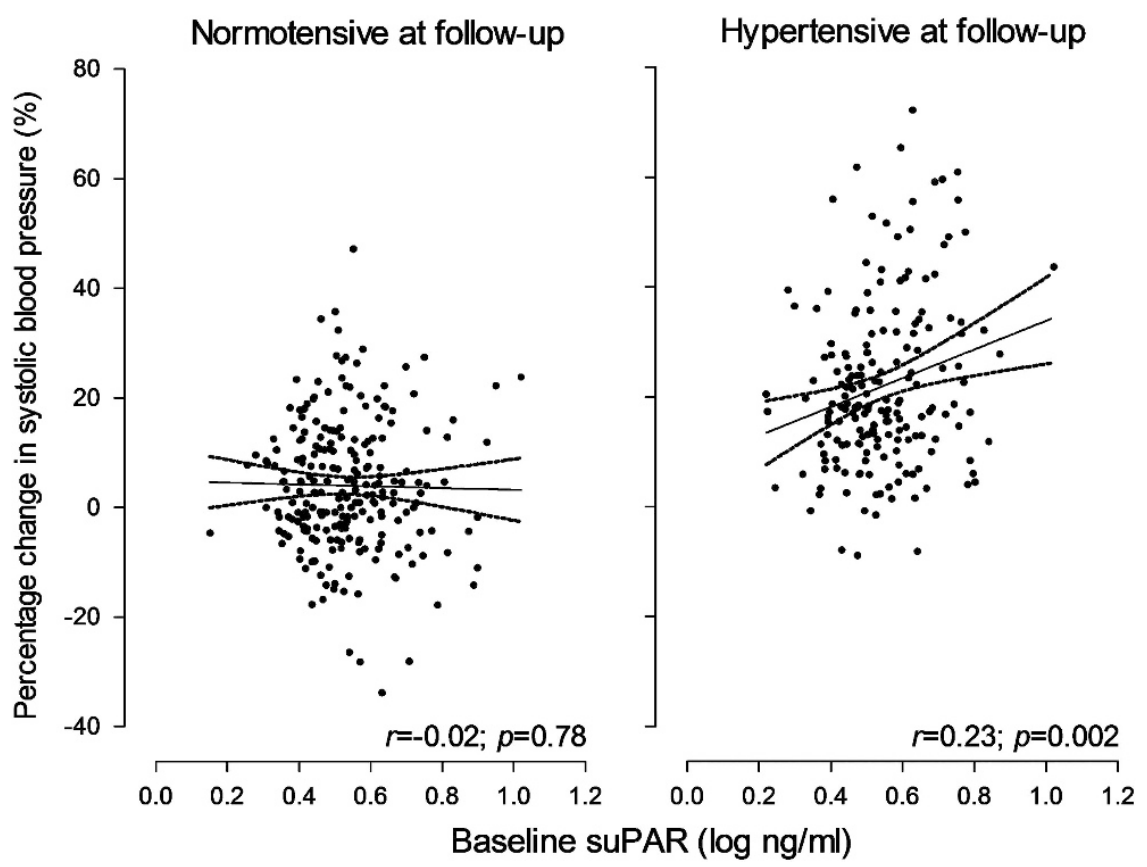

Figure 1 Single linear regression analyses of the percentage change in systolic blood pressure and baseline soluble urokinase plasminogen activator receptor (suPAR) in participants who remained normotensive and those who developed hypertension over five years, respectively.

Table 3 Independent associations with percentage change in systolic blood pressure

\begin{tabular}{|c|c|c|c|c|}
\hline \multirow[b]{3}{*}{ Multiple $R^{2}$; adjusted $R^{2}$} & \multicolumn{2}{|c|}{ Normotensive at follow-up } & \multicolumn{2}{|c|}{ Hypertensive at follow-up } \\
\hline & \multicolumn{2}{|c|}{$0.40 ; 0.38$} & \multicolumn{2}{|c|}{$0.39 ; 0.36$} \\
\hline & $\beta(95 \%$ Cl) & P-value & $\beta(95 \%$ Cl) & P-value \\
\hline Baseline SBP (mm Hg) & $-0.62(-0.73 ;-0.51)$ & $<0.001$ & $-0.56(-0.69 ;-0.43)$ & $<0.001$ \\
\hline Baseline suPAR (ng ml-1) & $-0.07(-0.18 ; 0.05)$ & 0.27 & $0.14(0.01 ; 0.28)$ & 0.043 \\
\hline Percentage change in suPAR (\%) & $-0.10(-0.22 ; 0.02)$ & 0.10 & $-0.06(-0.19 ; 0.07)$ & 0.36 \\
\hline Age (years) & & & $0.15(0.00 ; 0.29)$ & 0.050 \\
\hline Gender, male $(1,0)$ & $0.13(0.01 ; 0.24)$ & 0.030 & $0.11(-0.03 ; 0.25)$ & 0.12 \\
\hline Waist circumference $(\mathrm{cm})$ & $0.14(-0.01 ; 0.28)$ & 0.067 & $-0.14(-0.33 ; 0.06)$ & 0.18 \\
\hline Triglyceride:HDL-cholesterol ratio & $0.13(0.02 ; 0.25)$ & 0.026 & $0.17(0.03 ; 0.31)$ & 0.020 \\
\hline$\gamma$-Glutamyltransferase $\left(\mathrm{UI}^{-1}\right)$ & & & $-0.09(-0.23 ; 0.05)$ & 0.20 \\
\hline Creatinine clearance $\left(\mathrm{ml} \mathrm{min}^{-1}\right)$ & $-0.10(-0.23 ; 0.04)$ & 0.17 & $-0.03(-0.22 ; 0.16)$ & 0.74 \\
\hline
\end{tabular}

process of hypertension development or whether the hypertensive state may rather result in an increase in suPAR levels.

Inflammation affects multiple elements of the vascular wall, including the composition of the subendothelial matrix ${ }^{25}$ by mechanisms such as elastin and collagen turnover. ${ }^{26,27}$ uPAR controls basement membrane and extracellular matrix degradation, ${ }^{28}$ whereas CRP is involved in the process of vascular remodeling after injury. ${ }^{29}$ Inflammation may therefore have a role to decrease large artery vascular elasticity ${ }^{30,31}$ and alter endothelial function. ${ }^{32}$ The latter could lead to a decrease in nitric oxide bioavailability, ${ }^{33,34}$ impaired vasodilation and result in hypertension. ${ }^{34}$

On the other hand, higher blood pressure may be a stimulus for inflammation. ${ }^{35}$ Hypertension is associated with a prothrombotic state, which includes abnormal endothelial and platelet function, ${ }^{36}$ and evidently, treatment with antihypertensive drugs may have antifibrotic effects. ${ }^{37}$ Hypertension further links to vascular changes, which could lead to an imbalance of decreased nitric oxide production or increased reactive oxygen species production, promoting endothelial dysfunction ${ }^{4,38}$ and ultimately result in elevated suPAR. ${ }^{39}$ This is in concert with our findings where suPAR levels increased more in the group that became hypertensive than in those who remained normotensive.

It has been shown that CRP, a more familiar inflammatory marker, is correlated with SBP and that hypertension is associated with increased CRP where diabetes mellitus is apparent. ${ }^{40}$ On the contrary, in a study on 2432 apparently healthy subjects, Bautista et al. ${ }^{23}$ found that tumor necrosis factor- $\alpha$ and interleukin- 6 could be independent risk factors for hypertension, but did not find any association between CRP and hypertensive status. This was also the case in our study where the association with hypertension remained for suPAR, but not for 
Table 4 Logistic regression with hypertension status as dependent variable

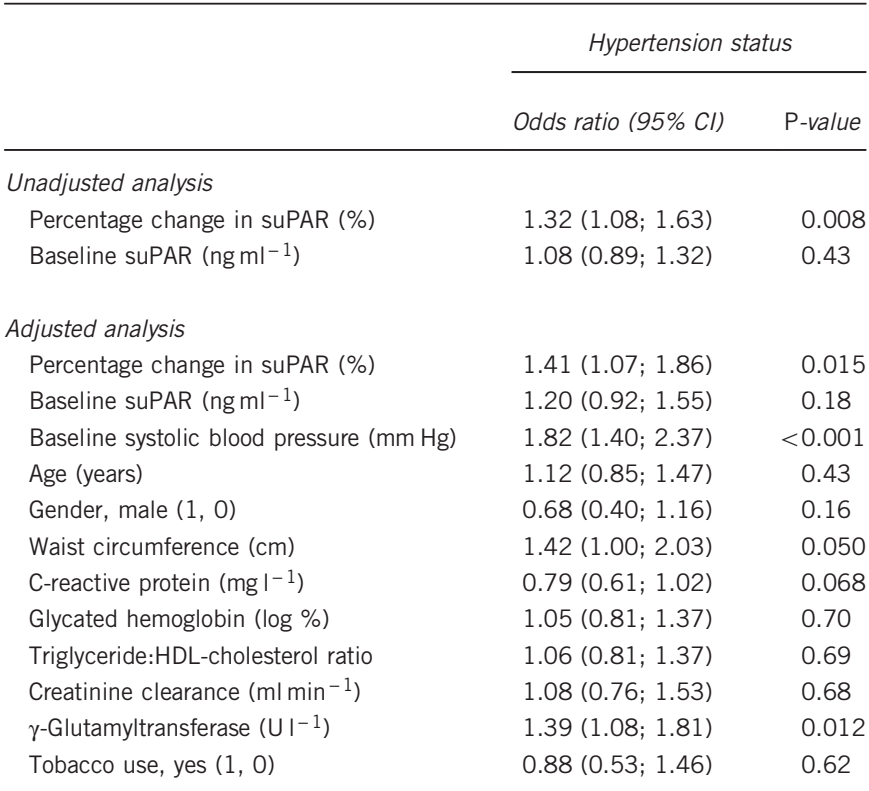

Abbreviations: $95 \% \mathrm{Cl}, 95 \%$ confidence intervals; HDL, high-density lipoprotein; suPAR, soluble urokinase plasminogen activator receptor.

Associations were determined by logistic regression analyses. $P \leqslant 0.05$ was regarded as statistically significant.

CRP when both markers were entered into the model. We further did not find any difference in CRP levels between hypertensives and normotensives at baseline or follow-up. Lyngbæk et al. ${ }^{39}$ showed that suPAR and CRP represent different biological processes in vascular inflammation. Where suPAR is secreted by endothelial and smooth muscle cells, ${ }^{6} \mathrm{CRP}$ originates from adipose tissue and is synthesized by the liver. ${ }^{39}$ This supports the association of CRP with inflammation related to adipose tissue, whereas suPAR may rather have a role as biomarker of inflammation in the vascular wall. ${ }^{39}$ Furthermore, Andersen et al. ${ }^{41}$ have indicated that suPAR may be a more stable marker of the immune state than CRP, as suPAR has a high stability in plasma samples, ${ }^{42,43}$ has limited circadian changes ${ }^{41}$ and is not affected by repeated freeze-thaw cycles of samples and sample schedule. ${ }^{43}$ These properties make suPAR an attractive clinical marker of inflammation, ${ }^{42}$ which could be of value in prognostic algorithms. ${ }^{44}$

This study adds information to the growing body of evidence that link inflammation to hypertension, also in Africans. ${ }^{22,23,45,46}$ However, to our knowledge, this is the first study to report a link between suPAR and hypertension in Africans. We included a relatively large sample of the understudied black South African population, and other studies on suPAR were mostly conducted on European populations. Nevertheless, this study must also be interpreted within the context of its potential limitations. As participants were recruited from one of the nine provinces of South Africa and only $21 \%$ were used at baseline, this study may not be representative of the entire black South African population. We did not measure body temperature or leukocyte count and can therefore not exclude underlying infections. As blood pressure was measured 5 years apart, we could not calculate the exact duration that each participant was hypertensive, and reverse causality should therefore be taken into consideration. However, the longitudinal nature of this study allowed for the evaluation of those who developed hypertension.

In conclusion, we showed that suPAR, an inflammatory marker, was higher and increased more prominently in participants who developed hypertension over 5 years than in those who remained normotensive. \%SBP was independently associated with baseline suPAR, whereas becoming hypertensive was associated with an increase in suPAR. Whether inflammation leads to the development of hypertension, or vice versa, remains to be established. However, it is known that suPAR is closely linked to cardiovascular disease. Our findings emphasize the important need to acknowledge the role of inflammation in hypertension and may permit the further investigation of the use of suPAR as a potential marker for early risk identification and intervention.

\section{CONFLICT OF INTEREST}

Jesper Eugen-Olsen is a founder, shareholder and board member of ViroGates A/S, Denmark, the company that produces the suPARnostic assay. JE-O is an inventor on a patent on suPAR and risk. Copenhagen University Hospital Hvidovre, Denmark, owns the patent, which is licensed to ViroGates A/S.

\section{ACKNOWLEDGEMENTS}

We are grateful towards the participants of this study, the PURE-SA research team, the field workers and supporting staff in the Africa Unit for Transdisciplinary Health Research (AUTHeR), North-West University, South Africa, as well as Dr S Yusuf (PURE-International) and the PURE project staff at the PHRI, Hamilton Health Sciences and McMaster University, ON, Canada. This work was financially supported by SANPAD (South Africa - Netherlands Research Program on Alternatives in Development), PHRI (Population Health Research Institute), the MRC (Medical Research Council) of South Africa, the South African NRF (National Research Foundation) (GUN numbers 2069139 and FA2006040700010), the North-West University, Roche Diagnostics and the German Academic Exchange Service (DAAD)-NRF. Opinions expressed and conclusions arrived at are those of the author and are not necessarily to be attributed to the DAAD-NRF.

1 Aznaouridis KA, Stefanadis Cl. Inflammation and arterial function. Artery Res 2007; 1: 32-38.

2 Harrison DG, Guzik TJ, Lob HE, Madhur MS, Marvar PJ, Thabet SR, Vinh A, Weyand CM. Inflammation, immunity, and hypertension. Hypertension 2011; 57: 132-140.

3 Sesso HD, Buring JE, Rifai N, Blake GJ, Gaziano JM, Ridker PM. C-reactive protein and the risk of developing hypertension. JAMA 2003; 290: 2945-2951.

4 Higashi Y, Kihara Y, Noma K. Endothelial dysfunction and hypertension in aging. Hypertens Res 2012; 35: 1039-1047.

5 Chen N-G, Abbasi F, Lamendola C, McLaughlin T, Cooke J, Tsao P, Reaven G. Mononuclear cell adherence to cultured endothelium is enhanced by hypertension and insulin resistance in healthy nondiabetic volunteers. Circulation 1999; 100: 940-943.

6 Intzilakis T, Hartmann G, Mouridsen MR, Eugen-Olsen J, Kumarathurai P, Madsbad S, Almdal TP, Haugaard SB, Sajadieh A. Soluble urokinase plasminogen activator receptor, C-reactive protein and triglyceride are associated with heart rate variability in non-diabetic Danes. Eur J Clin Invest 2013; 43: 457-468.

7 Eugen-Olsen J, Andersen O, Linneberg A, Ladelund S, Hansen TW, Langkilde A, Petersen J, Pielak T, Møller LN, Jeppesen J, Lyngbæk S, Fenger M, Olsen MH, Hildebrandt PR, Borch-Johnsen K, Jørgensen T, Haugaard SB. Circulating soluble urokinase plasminogen activator receptor predicts cancer, cardiovascular disease, diabetes and mortality in the general population. J Intern Med 2010; 268: 296-308.

8 Huai Q, Mazar AP, Kuo A, Parry GC, Shaw DE, Callahan J, Li Y, Yuan C, Bian C, Chen L. Structure of human urokinase plasminogen activator in complex with its receptor. Science 2006; 311: 656-659.

9 Theilade S, Lyngbæk S, Hansen TW, Eugen-Olsen J, Fenger M, Rossing P, Jeppesen JL. Soluble urokinase plasminogen activator receptor levels are elevated and associated with complications in patients with type 1 diabetes. J Intern Med 2014; 277: 362-371.

10 Harskamp RE, Roe MT. Soluble urokinase-type plasminogen activator receptor: a useful biomarker for coronary artery disease and clinical outcomes? J Am Heart Assoc 2014; 3: e001431.

11 Eapen DJ, Manocha P, Ghasemzedah N, Patel RS, Al Kassem H, Hammadah M, Veledar E, Le NA, Pielak T, Thorball CW. Soluble urokinase plasminogen activator receptor level is an independent predictor of the presence and severity of coronary artery disease and of future adverse events. J Am Heart Assoc 2014; 3: e001118.

12 Lyngbæk S, Marott JL, Sehestedt T, Hansen TW, Olsen MH, Andersen O, Linneberg A, Haugaard SB, Eugen-Olsen J, Hansen PR, Jeppesen J. Cardiovascular risk prediction in 
the general population with use of suPAR, CRP, and Framingham Risk Score. Int J Cardiol 2013; 167: 2904-2911.

13 Lloyd-Sherlock P, Beard J, Minicuci N, Ebrahim S, Chatterji S. Hypertension among older adults in low-and middle-income countries: prevalence, awareness and control. Int J Epidemiol 2014; 43: 116-128.

14 Teo K, Chow CK, Vaz M, Rangarajan S, Yusuf S. The Prospective Urban Rural Epidemiology (PURE) study: examining the impact of societal influences on chronic noncommunicable diseases in low-, middle-, and high-income countries. Am Heart $J$ 2009; 158: 1-7.

15 Schutte AE, Schutte R, Huisman HW, van Rooyen JM, Fourie CMT, Malan NT, Malan L, Mels CMC, Smith W, Moss SJ. Are behavioural risk factors to be blamed for the conversion from optimal blood pressure to hypertensive status in Black South Africans? A 5-year prospective study. Int J Epidemiol 2012; 41: 1114-1123.

16 Marfell-Jones M, Olds T, Stewart A, Carter L. International Standards for Anthropometric Assessment. Potchefstroom, South Africa: International Society for the Advancement of Kinanthropometry (ISAK), 2006.

17 Mancia G, Fagard R, Narkiewicz K, Redon J, Zanchetti A, Böhm M, Christiaens T, Cifkova R, De Backer G, Dominiczak A. ESH/ESC Guidelines for the management of arterial hypertension: The Task Force for the management of arterial hypertension of the European Society of Hypertension (ESH) and of the European Society of Cardiology (ESC). J Hypertens 2013; 31: 1281-1357.

18 ViroGates, suPARnostic. Enzyme Immunoassay for Quantitative Determination of Soluble Urokinase Plasminogen Activator Receptor in Human Plasma. Copenhagen Denmark, 2008.

19 Cockcroft DW, Gault MH. Prediction of creatinine clearance from serum creatinine. Nephron 1976; 16: 31-41.

20 Seedat YK, Rayner BL. South African Hypertension Guideline 2011. S Afr Med J 2011 102: $57-84$.

21 Pawlak K, Ulazka B, Mysliwiec M, Pawlak D. Vascular endothelial growth factor and UPA/suPAR system in early and advanced chronic kidney disease patients: a new link between angiogenesis and hyperfibrinolysis? Trans/ Res 2012; 160: 346-354.

22 Pruijm M, Vollenweider P, Mooser V, Paccaud F, Preisig M, Waeber G, Marques-Vidal P, Burnier M, Bochud M. Inflammatory markers and blood pressure: sex differences and the effect of fat mass in the CoLaus Study. J Hum Hypertens 2013; 27: 169-175.

23 Bautista L, Vera L, Arenas I, Gamarra G. Independent association between inflammatory markers (C-reactive protein, interleukin-6, and TNF- $\alpha$ ) and essential hypertension. J Hum Hypertens 2005; 19: 149-154.

24 Heijnen BF, Van Essen H, Schalkwijk CG, Janssen BJ, Struijker-Boudier HA. Renal inflammatory markers during the onset of hypertension in spontaneously hypertensive rats. Hypertens Res 2014; 37: 100-109.

25 Stehouwer C, Henry R, Ferreira I. Arterial stiffness in diabetes and the metabolic syndrome: a pathway to cardiovascular disease. Diabetologia 2008; 51: 527-539.

26 Yasmin SW, McEniery CM, Dakham Z, Pusalkar P, Maki-Petaja K, Ashby MJ, Cockcroft JR, Wilkinson IB. Matrix metalloproteinase-9 (MMP-9), MMP-2, and serum elastase activity are associated with systolic hypertension and arterial stiffness. Arterioscler Thromb Vasc Biol 2005; 25: 372-378.

27 Johnson C, Baugh R, Wilson C, Burns J. Age related changes in the tunica media of the vertebral artery: implications for the assessment of vessels injured by trauma. J Clin Pathol 2001; 54: 139-145.

28 Jain RK. Molecular regulation of vessel maturation. Nat Med 2003; 9: 685-693.

29 Hage F, McCrory M, Szalai A. C-reactive protein and cardiovascular disease: Lessons learned from studying genetically engineered mice. In: Nagasawa S (ed.) C-Reactive Protein-New Research. Hauppauge, NY: Nova Publishers. 2008.
30 Schillaci G, Pirro M. C-reactive protein in hypertension: clinical significance and predictive value. Nutr Metab Cardiovasc Dis 2006; 16: 500-508.

31 van Bussel BC, Schouten F, Henry RM, Schalkwijk CG, de Boer MR, Ferreira I, Smulders YM, Twisk JW, Stehouwer CD. Endothelial dysfunction and low-grade inflammation are associated with greater arterial stiffness over a 6 -year period. Hypertension 2011; 58: 588-595.

32 Zieman SJ, Melenovsky V, Kass DA. Mechanisms, pathophysiology, and therapy of arterial stiffness. Arterioscler Thromb Vasc Biol 2005; 25: 932-943.

33 Mahmud A, Feely J. Arterial stiffness is related to systemic inflammation in essential hypertension. Hypertension 2005; 46: 1118-1122.

34 Bautista LE. Inflammation, endothelial dysfunction, and the risk of high blood pressure: epidemiologic and biological evidence. J Hum Hypertens 2003; 17: 223-230.

35 Chae CU, Lee RT, Rifai N, Ridker PM. Blood pressure and inflammation in apparently healthy men. Hypertension 2001; 38: 399-403.

36 Sakamoto T, Kudoh T, Sakamoto K, Matsui K, Ogawa H. Antithrombotic effects of losartan in patients with hypertension complicated by atrial fibrillation: $4 \mathrm{~A}$ (Angiotensin II Antagonist of platelet Aggregation in patients with Atrial fibrillation), a pilot study. Hypertens Res 2014; 37: 513-518.

37 Mikami D, Kimura H, Kamiyama K, Torii K, Kasuno K, Takahashi N, Yoshida H, Iwano M. Telmisartan activates endogenous peroxisome proliferator-activated receptor- $\delta$ and may have anti-fibrotic effects in human mesangial cells. Hypertens Res 2014; 37 422-431.

38 Ceravolo R, Maio R, Pujia A, Sciacqua A, Ventura G, Costa MC, Sesti G, Perticone F. Pulse pressure and endothelial dysfunction in never-treated hypertensive patients. J Am Coll Cardiol 2003; 41: 1753-1758.

39 Lyngbæk S, Sehestedt T, Marott JL, Hansen TW, Olsen MH, Andersen O, Linneberg A Madsbad S, Haugaard SB, Eugen-Olsen J, Jeppesen J. CRP and suPAR are differently related to anthropometry and subclinical organ damage. Int J Cardiol 2013; 167. 781-785.

40 Rabkin SW, Langer A, Ur E, Calciu C-D, Leiter LA. Inflammatory biomarkers CRP, MCP-1, serum amyloid alpha and interleukin-18 in patients with HTN and dyslipidemia: impact of diabetes mellitus on metabolic syndrome and the effect of statin therapy. Hypertens Res 2013; 36: 550-558.

41 Andersen O, Eugen-Olsen J, Kofoed K, Iversen J, Haugaard SB. Soluble urokinase plasminogen activator receptor is a marker of dysmetabolism in HIV-infected patients receiving highly active antiretroviral therapy. J Med Virol 2008; 80: 209-216.

42 Thun $\varnothing$ M, Macho B, Eugen-Olsen J. suPAR: The molecular crystal ball. Dis Markers 2009; 27: 157-172.

43 Kofoed K, Schneider UV, Scheel T, Andersen O, Eugen-Olsen J. Development and validation of a multiplex add-on assay for sepsis biomarkers using XMAP technology. Clin Chem 2006; 52: 1284-1293.

44 Haupt TH, Petersen J, Ellekilde G, Klausen HH, Thorball CW, Eugen-Olsen J, Andersen O. Plasma suPAR levels are associated with mortality, admission time, and Charlson Comorbidity Index in the acutely admitted medical patient: a prospective observational study. Crit Care 2012; 16: R130.

45 Smith GD, Lawlor DA, Harbord R, Timpson N, Rumley A, Lowe GD, Day IN, Ebrahim S. Association of $\mathrm{C}$-reactive protein with blood pressure and hypertension life course confounding and Mendelian randomization tests of causality. Arterioscler Thromb Vasc Biol 2005; 25: 1051-1056.

46 Sesso HD, Wang L, Buring JE, Ridker PM, Gaziano JM. Comparison of interleukin- 6 and C-reactive protein for the risk of developing hypertension in women. Hypertension 2007; 49: 304-310.

Supplementary Information accompanies the paper on Hypertension Research website (http://www.nature.com/hr) 